\section{Relapsing-remitting painful ophthalmoplegia due to orbital myositis}

\author{
${ }^{1,2}$ E. Guaschino $(\triangle) \cdot{ }^{1} \mathrm{C}$. Zandrini • ${ }^{1}$ G. Minonzio \\ ${ }^{1}$ A. Mancioli • ${ }^{1}$ M. Broggini • ${ }^{1}$ G. Bono \\ ${ }^{1}$ Departments of Clinical Medicine \\ and Diagnostic Imaging, \\ Units of Neurology and Neuroradiology, \\ Ospedale di Circolo, \\ Università dell'Insubria, Varese, Italy \\ ${ }^{2}$ U.O. Neurologia, \\ Ospedale di Circolo, \\ Viale Borri 57, I-21100 Varese, Italy \\ e-mail: neurova@tin.it \\ Tel.: +39-0332-393184 \\ Fax: +39-0332-393184
}

Published online: 15 December 2005

\begin{abstract}
We describe a woman with a long history of relapsing-remitting painful ophthalmoplegia in a seasonal pattern, due to an isolated orbital myositis that was - at our observation - classified in the context of the so-called SAPHO syndrome. She had been previously treated with corticosteroid therapy, but the association with immunosuppressive drugs produced a more prompt resolution of both the headache and ophthalmoplegia, and of the magnetic resonance imaging pictures of isolated myositis as well.
\end{abstract}

Keywords Painful ophthemolplegia - Orbital myositis • SAPHO syndrome

In the original IHS Classification [1], Ophthalmoplegic migraine was defined as a migraine headache (1.3) overlapping with paresis of one or more ocular nerves, in the absence of demonstrable intracranial parasellar lesions. In the additional "comment", based on the frequent longlasting course of symptoms (a week or more) in many cases, a relationship to the syndrome rather than to true migraine was stressed. In the ICHD-II [2], Ophthalmoplegic "migraine" has now been moved to the chapter of "Cranial neuralgias", an entity (13.17) separate from the Tolosa-Hunt syndrome (13.16) and characterized by migraine-like headache accompanied or followed - within the strict limit of 4 days - by paresis of oculomotor nerves (commonly the third nerve).

The present report refers to a 41-year-old woman who came to our observation for chronically recurring and long-lasting episodes of migraine-like headache, accom- panied by variable degrees of diplopia due to constantly unilateral oculomotor paresis affecting abduction movements of the left eye.

Her history started at the age of 18 years old: recurring migraine-like attacks with bilateral fronto-orbital pain becoming chronic and more or less continuous within days, and with disabling phases at times, were occurring almost every year in active periods lasting 2-3 months. Headache was accompanied by ipsilateral conjunctival injection, eye-lid oedema and diplopia (with onset in the first to second week and spontaneous remission usually days or weeks after headache resolution). Since the onset there had been only one single long-lasting headache-free period of 10 years. During the last seven years the episodes returned to a regular circa-annual presentation with seasonal (spring-time) rhythmicity.

Only the last two episodes were treated with systemic corticosteroids, which produced remission (within 4-6 weeks) of the daily orbitofrontal headache without significant effects on the duration of diplopia, which lasted more or less as long as in the previous - untreated episodes (2-3 months).

The first RMN investigation performed within 3 weeks of symptom onset (last episode) gave normal results without evidence of orbital and intracranial pathology. Only 6-8 weeks after full development of both headache and ophthalmoplegia RMN showed an isolated orbital myositis affecting the left lateral rectus. She was already under steroid treatment and indomethacin $75 \mathrm{mg}$ /day for 7 days was able to induce full recovery of headache.

A systematic evaluation of local and systemic inflammatory conditions was carried out with negative results. A clinical history, however, disclosed that she had been suffering from psoriasis, palmoplantar pustulosis (PPP), isolated episodes of arthritis and probably osteitis, thus suggesting SAPHO Syndrome (synovitis, acne, pustulosis, hyperostosis, osteitis) [3]. Therefore immunosuppressive treatment was added to corticosteroids with a progressive reduction of diplopia within the following 4 weeks and the subsequent resolution of the RMN pictures of isolated myositis.

Isolated, subacute, chronic myositis may produce symptoms resembling ophthalmoplegic migraine and Tolosa-Hunt syndrome. The association with systemic autoimmune disorders should be considered. In the early stages or in the acute forms, RMN may be negative and follow-up studies are needed.

\section{References}

1. Headache Classification Committee of the International Headache Society. (1988) Classification and diagnostic criteria for headache disorders, cranial neuralgias and facial pain. Cephalalgia 8[Suppl 7]:1-96 
2. Headache Classification Subcommittee of the international Headache Society (2004) The International Classification of Headache Disorder, 2nd Edn. Cephalalgia 24:1-160
3. Ajitsaria R, Dale R, Ferguson V, Mayou S, Cavanagh N (2001) Psoriasis, psoriatic arthropathy and relapsing orbital myositis. Clin Exp Dermatol 26(3):274-275 